\title{
Basketball Activity Recognition using Wearable Inertial Measurement Units
}

\author{
Le Nguyen Ngu Nguyen \\ Universitat Politècnica de Catalunya \\ - BarcelonaTech \\ Rambla de l'Exposició 59-69, \\ Vilanova i la Geltrú, Spain \\ Queen Mary University of London \\ Mile End Road, E1 4NS, London, UK \\ le.nguyen.ngu.nguyen@upc.edu
}

\author{
Daniel Rodríguez-Martín \\ Technical Research Centre for \\ Dependency Care and Autonomous \\ Living \\ Universitat Politècnica de Catalunya \\ - BarcelonaTech \\ Rambla de l'Exposició 59-69, \\ Vilanova i la Geltrú, Spain \\ daniel.rodriguez-martin@upc.edu
}

\author{
Andreu Català \\ Technical Research Centre for \\ Dependency Care and Autonomous \\ Living \\ Universitat Politècnica de Catalunya \\ - BarcelonaTech \\ Rambla de l'Exposició 59-69, \\ Vilanova i la Geltrú, Spain \\ andreu.catala@upc.edu
}

\author{
Carlos Pérez-López \\ Universitat Politècnica de Catalunya \\ - BarcelonaTech \\ Technical Research Centre for \\ Dependency Care and Autonomous \\ Living \\ Rambla de l'Exposició 59-69, \\ Vilanova i la Geltrú, Spain \\ carlos.perez-lopez@upc.edu
}

\author{
Albert Samà \\ Universitat Politècnica de Catalunya \\ - BarcelonaTech \\ Technical Research Centre for \\ Dependency Care and Autonomous \\ Living \\ Rambla de l'Exposició 59-69, \\ Vilanova i la Geltrú, Spain \\ albert.sama@upc.edu
}

\author{
Andrea Cavallaro \\ Queen Mary University of London \\ Mile End Road, E1 4NS, London, UK \\ a.cavallaro@qmul.ac.uk
}

\begin{abstract}
Analysis of human movement is a growing research area within the field of sport monitoring which aims to enhance the performance of athletes, predicting injuries or optimizing training programs. Camera-based techniques are the most spread method to evaluate although frequently this method can be cumbersome and, furthermore, specific movements where performance is analyzed are not possible to distinguish. Wearable inertial systems however, are capable to ameliorate this matter.

This paper presents a new wearable sensing system with the aim to record human movements in the field of sport. A new paradigm is presented with the purpose of monitoring basketball players with multiple inertial measurement units. A data plan collection has been designed and experimental results show the potential ability of the system in basketball activity recognition.
\end{abstract}

\section{Categories and Subject Descriptors}

H.1.2 [User/Machine Systems]: User/Machine Systems - Human factors, Human information processing.

\section{General Terms}

Algorithms, Design, Experimentation, Human Factors.

\section{Keywords}

Wearable computing, Activity recognition, Sport.

\section{INTRODUCTION}

In recent years, human activity recognition has facilitated novel applications in various domains, including healthcare, life monitoring, entertainment, and sport [1]. Its main goal is to identify the activities of one person or a group of agents from observations acquired by sensing devices such as wearable sensors and ambient cameras.

Traditionally, vision systems using external cameras have been deployed to collect information of human activities. Researchers in computer vision have reached a large number of achievements in human activity analysis [1]. They are widely applied in surveillance systems and human-computer interaction applications. However, the vision-based approach suffers from issues related to obtrusiveness and complexity of real-world settings. They may violate users' privacy and their coverage is restricted in specific spaces. Due to technical characteristics of cameras, their performance is effected by occlusion and cluttered scenes.

Recently, advances in sensor technology allow deploying wearable devices in human activity analysis. They have enabled continuous long-term activity monitoring beyond instrumented areas. Moreover, the sensors embedded in these devices, such as accelerometers and gyroscopes, collect signals directly from body movements, in lieu of inferring from visual data. On the other hand, wearable systems have difficulty in gathering observations on interaction between multiple agents. Thus, there appears a new research trend that combines those two types of sensing in order 
to overcome the above limitations. It also raises challenging issues on synchronization, association, and fusion of multimodal data.

Sport is a potential application domain for human activity analysis. Understanding athletic movements helps coaches and managers to evaluate player's performance. It provides analytic information to predict injuries, optimize training programs, and support strategic decision making. To do that, a sport activity analysis system can identify actions and extract features of movements (e.g. motion intensity, speed...). The applications can be customized for each specific sport and exploit expert knowledge.

The problem of sport activity analysis shares common issues with the general one [2]. They are:

- Intra-class variability: The same activity may be performed in different ways, depending on internal status of the player. For example, a basketball player moves slowly at the end of the match due to fatigue. Furthermore, each individual player has his or her own styles of movements.

- Inter-class similarity: Some activities are semantically different but produce similar characteristics in inertial sensing data. The combination of multiple modalities (e.g. cameras and wearable inertial sensors) can help to eliminate the ambiguity.

- $\quad$ The NULL class problem: Not all inertial sensing data are necessary for sport activity analysis. The existence of irrelevant activities (so-called NULL class) makes confusion for activity classification algorithms. Modelling the NULL class is not possible because it represents the set of infinite arbitrary activities.

In addition, sport activity analysis has its own challenges, including:

- Definition of relevant activities: The system is only possible to model and analyze a limited set of activities, which should be proposed by sport experts. This requires collaboration between two groups of scientists whose mindset is different. Moreover, human activities contain spatial and temporal constraints, which should be considered when modelling.

- Class imbalance: The players do not perform every activity in the same duration and number of repetitions. For instance, a soccer striker spends most of the time moving (e.g. walking and running) while he/ she only shoots few times in a match.

- Data annotation: In order to train a supervised human activity analysis system, we need to collect a significant amount of data and annotate the datasets. The process is time-consuming and requires being extremely accurate. The difficulty increases in case of team sport, where multiple players interact with each other.

- Sensing data characteristics: Movements in a competitive match are much faster than those in daily living activities. The variation of inertial systems data is, then, much higher. Thus, parameters for the algorithms should be modified relying on characteristics for each type of sport. Sensor design requirements should change according to this issue (e.g. acceleration in sport has larger range than in monitoring systems for daily living activities or patients with chronic diseases).

In this paper, a new sensor design is introduced with the aim of recording and recognizing athletic movements in basketball. Basic basketball specific movements such as jumps, lateral displacements, forward/backward moves and body rotations (pivot) are evaluated. Sport dependent and meaningful actions (dribbling, shooting, etc) are also considered. The developed device is attached on each athlete's body and limbs establishing a multiple sensory system. Features are extracted from accelerometers and the SVM-based classification algorithm is used to recognize the activities.

The rest of this paper is organized as follows. Section 2 reviews related work of using wearable sensors in sport. The sensor system is introduced in Section 3. Then, the data collection session and activity recognition method are described in Section 4 and Section 5, respectively. The experimental results and analysis are presented in Section 6. Finally, Section 7 concludes our paper.

\section{RELATED WORK}

In recent years, activity monitoring, performance evaluation and providing feedback in sport have been deployed with the use of wearable systems. Multiple types of sensors have been used: accelerometers, gyroscopes, pressure sensors, heart-rate monitor, etc. The users could wear one or more sensors on different locations of their bodies. Lara and Labrador presented a comprehensive survey on human activity recognition systems using wearable devices [3]. The authors depicted the system pipeline and described principle techniques which included feature extraction, classification algorithms and qualitative evaluation. Open relevant problems were also introduced: standardized datasets, highly-complex activities, extraction of activity characteristics, participatory sensing and early prediction of human activities. Following, some relevant papers within the field of sports employing wearable systems are reported.

Regarding specific activity recognition within the field of sports there are several works which analyze particular movements for different sports. For example, Kelly et al. studied techniques to automatically identify tackles and collisions in rugby [4]. Their experimental device, consisting of a GPS receiver and an accelerometer, was placed between the shoulder blades overlying the upper thoracic spine of each athlete. Support vector machine (SVM) and hidden conditional random field (HCRF) were applied to detect collision events. The classification results were then combined with AdaBoost. The recall and precision of their proposed system were $93.3 \%$ and $95.8 \%$, respectively. On the other hand, Morris et al. used inertial sensors to monitor exercise activities [5]. The authors first discriminate exercise and non-exercise movements. Then, they recognize and count the repetition of activities. Their method achieved the accuracy of $96 \%$ when recognizing 13 exercise actions.

Bächlin et al. [6] presented a wearable assistant for swimmers, called SwimMaster. With its acceleration sensors and feedback devices, the system extracts swim parameters and performance assessment in real time. The authors organized different scenarios to evaluate body measurement (angle, rotation, and balance), performance parameters, and feedback effectiveness. 
Harle et al. [7] built an on-body sensing system to monitor the performance in sprinting training sessions. Force sensitive resistors are embedded in athletes' shoes to collect pressure data, which were then analyzed and presented to the coach. The accuracy of their proposed system reached millisecond level in estimating ground contact time.

Aiming to replicate the role of expert evaluations in climbing sport, Ladha et al. [8] developed ClimbAX, which was a wearable acceleration sensing platform to record climber's movements. The captured data were analyzed to extract human movements and climbing sessions. After that, the assessment parameters were extracted, including: power, control, stability and speed. Their system was extensively experimented with 53 climbers under competition scenarios and could produce scores that strongly correlated with official expert results.

Furthermore, there are some works which provide a feedback to the athlete with the aim of giving an stimulus and, then, ameliorate the performance. Bächlin et al. used LED lights to provide real-time feedback to the swimmers [6]. In contrast, the system of Velloso et al. has a feedback mechanism that helps users to manipulate their movements in weightlifting [9].

Another important work was from Ghasemzadeh and Jafari who collected physiological data from a body sensor network to provide corrective feedback for baseball players [10]. They interpreted complex movements to generate motion transcripts which were used for measuring coordination among limb segments and joints.

\section{SENSOR DESIGN FOR BASKETBALL}

A new sensor system, called BSK board has been developed at the Technical Research Centre for Dependency Care and Autonomous Living (CETpD) to record data of particular movements in basketball. The BSK board is an inertial measurement unit (IMU) with the aim of capturing inertial data that requires a long range of acceleration as well as experiments requiring barometric information. It has a size of $62 \times 35 \times 24 \mathrm{~mm}^{3}$ and weighs $38 \mathrm{~g}, 62 \mathrm{~g}$ with battery. The BSK hardware structure is similar to $9 \times 2$ IMU but with new devices aimed to reduce the energy consumption and increase computational capacity [11].

This board is a development tool, which includes inertial sensors, a storage unit and a small interface in order to send and receive commands. The BSK board has 4 main parts, the power system, the $\mu \mathrm{C}$ and its interface, the analog system and the communications module. Figure 1 shows the main scheme of the circuit. The system is powered with two AAA standard batteries. The average consumption of the system ( 7 tests of five-minutes each) is $56.73 \pm 0.21 \mathrm{~mA}$, having an autonomy of more than 20 hours in a $1200 \mathrm{mAh}$ battery at 3 Volts. With a standard alkaline battery (750mAh), autonomy could be about 13.2 hours.

The power distribution is comprised with 4 regulators. The digital regulator, the analog regulator, the comm regulator and the backup regulator. The digital regulator provides power to the digital system (microcontroller, inertial sensors I/O, $\mu$ SD Card, and USB interface). The analog regulator supplies power to the inertial sensors, being separated and isolated from the rest of the circuit by means of an own ground plane. The comm regulator supplies voltage to the Bluetooth module. This device can consume more than $40 \mathrm{~mA}$ alone, for this reason it should be isolated from the rest of the circuit in order to avoid peaks of currents that affects the voltage stability at the analog or digital system. Finally, the backup regulator keeps a regulated voltage to the $\mu \mathrm{C}$ 's backup system and the real-time clock system.

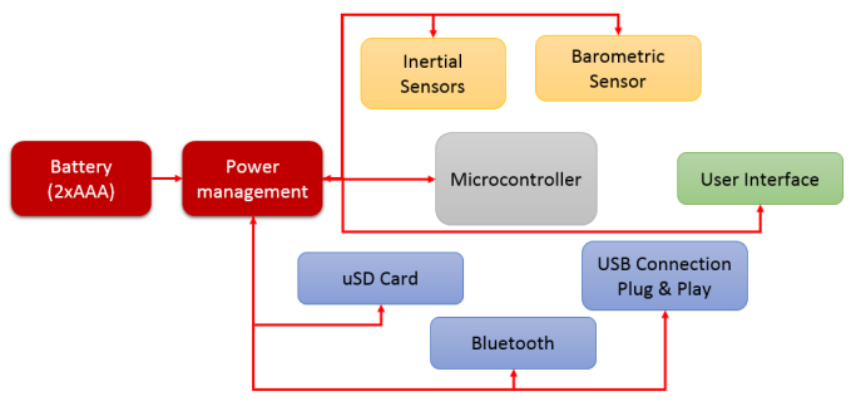

Figure 1. Main BSK board structure

\subsection{BSK Microcontroller}

The microcontroller $(\mu \mathrm{C})$ that manages the internal processes from the BSK board is a STM32F415 from STMicroelectronics. This microcontroller is a Cortex ${ }^{\mathrm{TM}}-\mathrm{M} 4$ CPU with floating point unit, which lets computing advanced online algorithms. The maximum speed of this device is $168 \mathrm{Mhz}$ and contains $1 \mathrm{MB}$ flash memory and $192 \mathrm{~KB}$ of RAM, compared to the $128 \mathrm{~KB}$ and $16 \mathrm{~KB}$, respectively, from the $9 \times 2$ 's $\mu \mathrm{C}$. The BSK board contains two external clocks, one to run the internal oscillator circuit and a Real-Time Clock to count seconds with high accuracy. One of the main features of this $\mu \mathrm{C}$ is the Direct Memory Access (DMA), which is able to exchange data among the different peripherals and between the peripherals and the $\mu \mathrm{C}$. Finally, the STM32F4 includes up to 15 communication interfaces among which UART, SPI, $\mathrm{I}^{2} \mathrm{C}$, SDIO and USB 2.0 full-speed On-The-Go controller are of main importance. The $\mathrm{BSK} \mu \mathrm{C}$ is a 64 pin device with debug mode able to enter in different low-power modes, in order to increase the autonomy of the BSK board. With the peripherals configured to manage the BSK board. Figure 2 shows the expected consumption at different work modes.

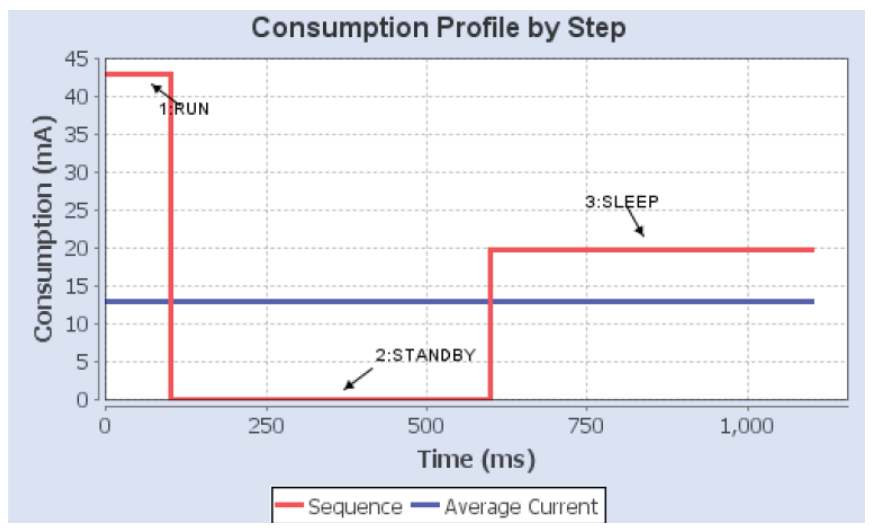

Figure $2 . \mu \mathrm{C}$ average consumption in different work modes

\subsection{Embedded Sensors}

The BSK board contains different MEMS sensors, one the one hand it includes the LSM9DS0, a System in Package (SiP) which contains an Accelerometer + Magnetometer system and a Gyroscope system (Figure 3). On the other hand the BSK board includes a pressure sensors as well in order to detect fallings or movements with a change of altitude (mainly postural transitions)..

The LSM9DS0 MEMS provide different interruptions in order to first notify the $\mu \mathrm{C}$ when data is ready to be read with a configured 
output data rate and, second, provide interrupts when a configured threshold is surpassed with the aim of awaken the $\mu \mathrm{C}$ just in case enough movement is detected. This mode of work allows to save much power analyzing data of weak importance such a static movements (sit, stand or lying).

The MEMS pressure sensor is a barometric sensor. The main feature is the RMS noise, which is $0.02 \mathrm{mbar}$. It is considered that the traveled distance of the trunk in a Sit to Stand or Stand to Sit Posture Transition is $6.1 \mathrm{~Pa}$ or $0.061 \mathrm{mbar}$ [12]. This means that according to LPS331AP's RMS noise minimum value, a Posture Transition can be detected. Furthermore, fallings, going up/down stairs or elevators could be also detected. For this reason, the MEMS pressure sensor is an interesting tool to be added at any human activity recognition system.

The LPS331AP offers an ODR up to $25 \mathrm{~Hz}$, although the minimum RMS noise can only be achieved with $12.5 \mathrm{~Hz}$. According to Zhou et al. this is a low frequency to catch all human movements [13], however, and having into account that Posture Transitions [14] and Walking Bands are below this frequency [1], $12.5 \mathrm{~Hz}$ is enough to identify all these activities.

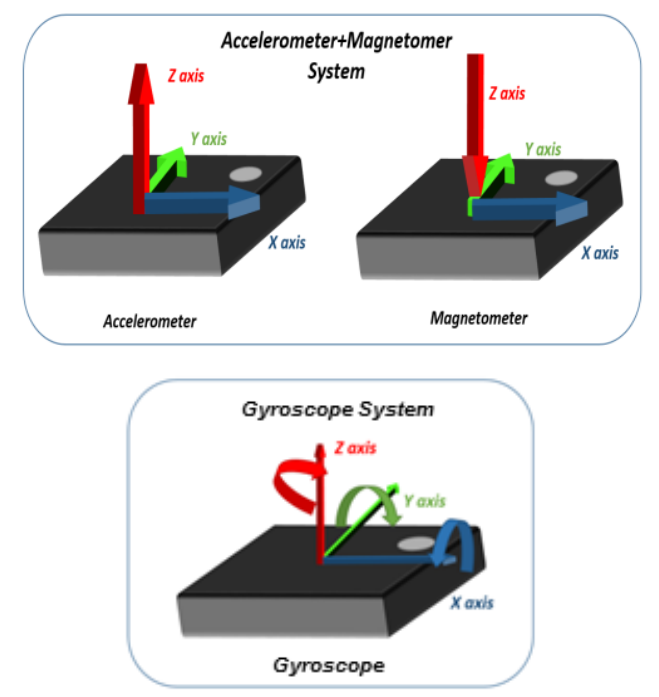

Figure 3. BSK board inertial MEMS (LSM9DS0)

\subsection{Communication Component}

The BSK board has been thought to download the obtained data without removing any piece of the device. In other words, this design has been performed with the aim of being able to download data through USB connection.

Data is stored in a $\mu$ SD card by means of the SDIO interface and with FAT32 system file format allowing to store much more data than the 2GB allowed by FAT16. The device contains a specific hinge socket where the $\mu \mathrm{SD}$ card is inserted. This socket does not allow the $\mu \mathrm{SD}$ card to move in aggressive execution tasks such as sprinting or jumping, avoiding then communication errors.

The Full-Speed On-the-Go USB system has been incorporated to the BSK board including a USB buffer device, which filters the noise and allows to isolate electromagnetically the BSK USB circuitry from PC circuitry in order to ensure a robust communication between the two devices. When the BSK board is plugged to PC, a pop-up window appears in the screen notifying the user that a device has been connected and is ready to be read being able, then, to download all data to the desired destination folder.

\section{DATA COLLECTION}

The BSK board has been employed to perform the specific database. The sampling frequency is $200 \mathrm{~Hz}$ and it collects data from accelerometer, gyroscope, magnetometer, temperature and barometric sensor. The five sensors are attached to body as shown Figure 5.

The test protocol consist of 10 different activities executed continuously which includes a jump at the beginning and at the end of each activity and a standing between the different series. Regarding to basketball movements, these are the following activities: walking, running, jogging, pivot (rotation with one fixed foot), shoots from different locations, layups, sliding and sprinting. We also use one more label, undefined, to annotate irrelevant movements.

The dataset activities are repeated different times in order to obtain more reliable and evaluable data and the test is videorecorded in order to have a ground-truth for annotation. Since there may be points of view that camera cannot properly capture, an extra camera has been included in a side of the basketball court as shown in Figure 4.

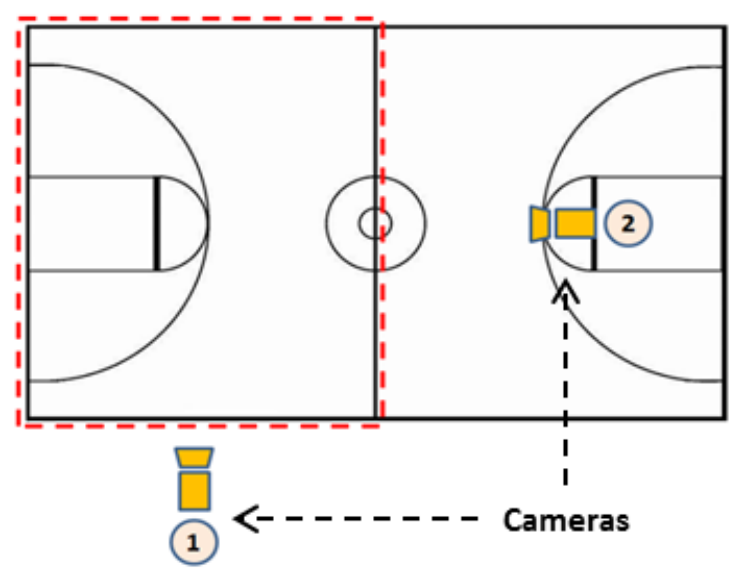

Figure 4. Layout of the cameras on the basketball court

At the beginning and at the end of each user data collection, a visual event is performed in order to synchronize cameras and inertial systems. Then after data collection data integrity is checked, inertial signal is synchronized with video signal and, then, labeled according to recorded video. We propose two options:

- Sensor falling: When the device falls down, the embedded accelerometer generates a peak and change of axes. However, this step must be performed before attaching the sensors to human body. Thus, there is a significant amount of unusable signals in the recorded data.

- Jumping action: Similarly, when the subject jumps, a sudden change appears in acceleration signals. This can be used as a symbol to match the event on inertial signals with that on videos. The limitation is jumping is one of our activities-of-interest. Therefore, the annotator should be careful when finding the event.

The data collection plan is required to contain the activities-ofinterest in a reasonable order so that it is feasible for the subjects 
to perform the activities (i.e. not feeling exhausted). Our data collection plan is designed with the consultation of sport experts and amateur basketball players.
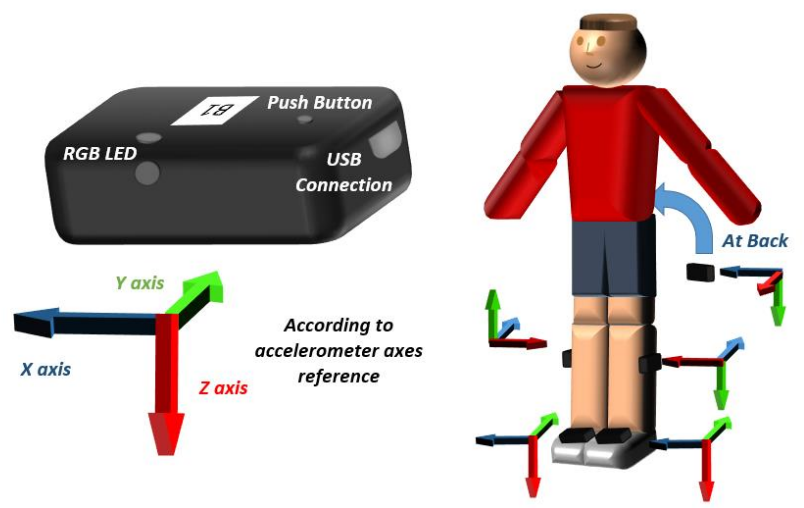

Figure 5. BSK board model and sensors set up

\section{BASKETBALL ACTIVITY RECOGNITION}

After collecting data on movements in basketball, we analyze the inertial signals to recognize the activities. In this paper, our objective is to classify basic actions in basketball, including: walking, jogging, running, sprinting, jumping, jumpshot, layupshot and pivot. In the experiments, we only use data recorded by two accelerometers on player's feet to recognize these movements.

Our activity recognition method has five steps: (i) preprocessing, (ii) segmentation, (iii) feature extraction, (iv) standing - moving separation, and (v) moving activity recognition (Figure 6).

First, preprocessing techniques are performed on inertial signals, including downsampling and filtering. Because the original data is sampled at $200 \mathrm{~Hz}$, it may contain redundant information. Thus, we downsample both accelerometers to $40 \mathrm{~Hz}$. That sampling frequency is selected to balance between recognition accuracy and computational cost [13]. After that, those signals are passed through a low-pass filter with $15 \mathrm{~Hz}$ as the cut-off frequency.

Then, the inertial signals are divided into equal segments whose duration is called window length. With the aim of not losing inertial events between windows, the windows are overlapped at $50 \%$. Features are then extracted from each segment and the classification algorithm produces one label for each segment.

At the third step, we use a simple feature to separate standing from moving sessions. All standing segments are removed and the remaining data is used to recognize moving activities (e.g. running, jumping, pivot, etc).

Finally, features that can contain information in time domain, frequency domain, and correlation between the sensors, are extracted. They are fed to the activity recognition algorithm.

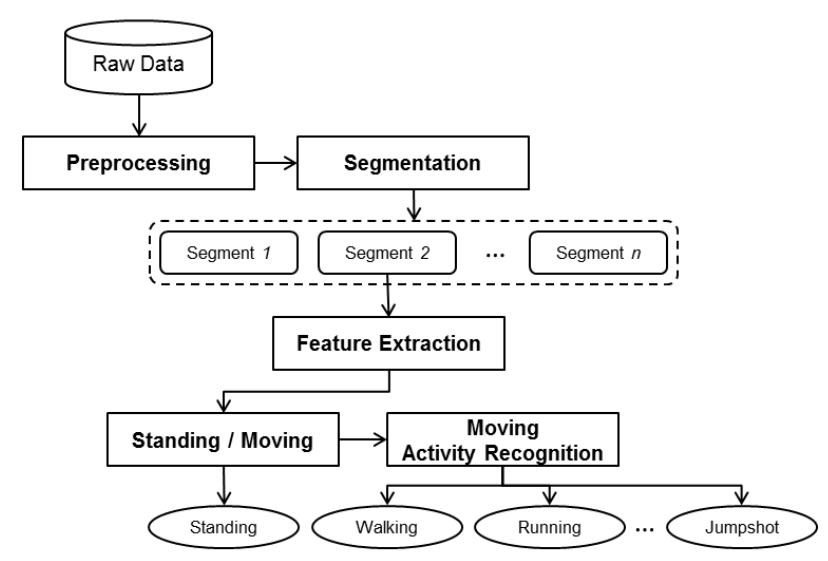

Figure 6. Our proposed activity recognition algorithm

\subsection{Feature Extraction}

In each segment of inertial data, we extract different types of features. We employ two accelerometers on the feet, this is to say, three axes for each accelerometer. Then, the root mean square value for each accelerometer is calculated. Thus, in each sample of each accelerometer, four values (three raw values and one amplitude value) are obtained. From each of them, time and frequency domain features are extracted, including: range, sum, mean, standard variation, mean crossing rate, skewness, kurtosis, frequency bands, energy, and number of peaks above a threshold.

Moreover, correlation between three axes of each sensor and each pair of axes on two sensors are also considered. To do so, correlation coefficients between each pair of axes on each sensor are computed. Then, the same computation on each pair of respective axes on two sensors is performed.

\subsection{Activity Recognition Algorithm}

Based on observations, standing and other activities can be discriminated based on the range values of acceleration on the Zaxis. In each segment, the difference between maximum and minimum values of acceleration, which is called range, is calculated. If the value is higher than an optimized threshold, we classify the segment as moving; otherwise, it is a standing segment. We trained a linear SVM classifier on range values to estimate this threshold. Hence, it can be modified for different datasets.

After removing all standing segments, features described in Section 5.1 employed to recognize moving activities of basketball players are extracted. Each segment of inertial signals becomes a feature vector or sample of the classifier. We feed them to the classification algorithm for training and testing in two cases, which are called same-person and cross-person. In the former, we randomly select samples for training and testing from the same player. In the later, we train the recognition algorithm on a group of players and test it on the other one. In both cases, training and testing datasets are different. However, the second one is more challenging because players with distinct physical characteristics generate different inertial data, even when they perform the same activities.

\section{EXPERIMENTAL RESULTS}

In our experiments, we set the window length to 128 raw samples and the overlapping percentage to $50 \%$. That means the duration of each segment is 3.2 seconds. Then, we split the samples into training and testing datasets according to two cases defined in 
Section 5.2. Our dataset contains inertial signals of three subjects. Thus, in the cross-person setting, we use data from two players for training and one for testing. The final result is the average measures from all splitting configurations of subjects. This can be considered as leave-one-subject-out evaluation strategy. We use LibSVM library [16] to implement the multi-class SVM classifier.

In Figure 7, the actual and predicted labels of standing and moving of one player are depicted. Most of confusion appears when the player performs the pivot action. In this action, one leg of the player is kept stable and the other can moves. Thus, it generates similar signals to standing in one foot. The threshold for separating standing and moving is 4 and the accuracy is $92 \%$.
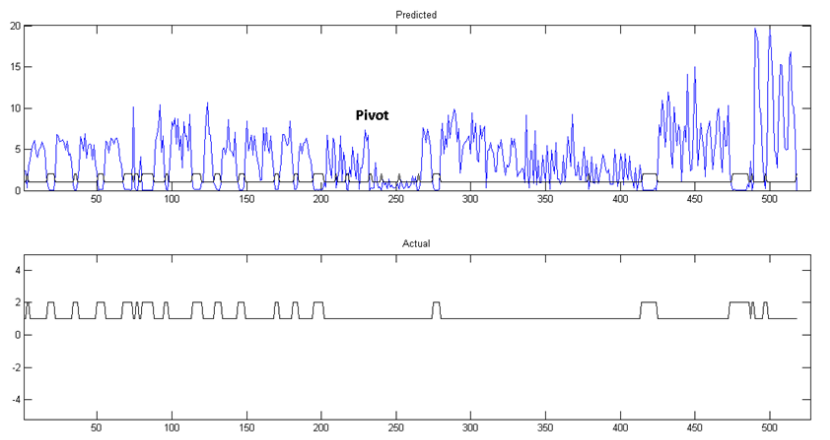

Figure 7. Actual and predicted labels of standing and moving. The blue plot is the range value of $\mathrm{Z}$-axis acceleration (best view in color).

Then, the moving activities are divided into two sets, namely step and jump activities. The step-related activities include walking, jogging, running, and sprinting while the jump-related activities contain jumping, layupshot, and jumpshot. Figure 8 shows the confusion matrices in both evaluation cases. It is possible to clearly discriminate step-related activities on the same player. Nevertheless, due to distinction in physical characteristics, two players perform the same activities in different ways. Therefore, in the cross-person evaluation setting, confusion appears in both step-related jump-related activities and the precision decreases. In the case of jump-related activities, all of them include a jumping action. Consequently, they produce similar inertial data.

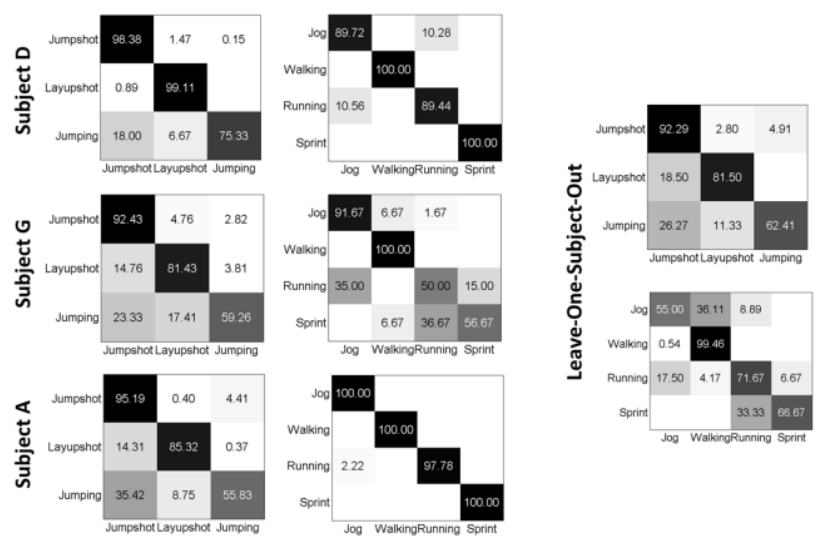

Figure 8. Confusion matrices of activity recognition in two sets (step-related and jump-related)

Figure 9 illustrates the performance of our activity recognition algorithm on all activities (walking, jogging, running, sprinting, jumping, jumpshot, layupshot and pivot) in cross-person setting. Similar confusion is revealed when we train and test the classification model on different players. Furthermore, when performing layupshot, the player dribbles then throws the ball to the basket. Dribbling (i.e. running with ball), generates acceleration signals which are similar to those in jogging or running. This motivates to integrate sensors in other body parts (e.g. on the wrists) to distinguish these activities.

\begin{tabular}{|c|c|c|c|c|c|c|c|c|}
\hline Jog & 51.98 & 30.39 & 3.92 & 11.86 & & & 1.85 & \\
\hline Walk & 1.25 & 80.98 & 15.65 & 1.15 & 0.64 & 0.32 & & \\
\hline Jumpshot & & 10.87 & 78.65 & 2.25 & 2.86 & 5.38 & & \\
\hline Layupshot & 3.10 & 17.23 & 9.89 & 65.39 & 1.28 & & 3.10 & \\
\hline Pivot & & & 6.94 & & 90.22 & 2.84 & & \\
\hline Jumping & & 5.40 & 15.28 & 7.47 & 2.78 & 66.60 & 2.47 & \\
\hline Running & 11.11 & 7.04 & & 27.96 & & & 44.63 & 9.26 \\
\hline \multirow[t]{2}{*}{ Sprint } & & & & & 11.43 & 4.17 & 25.12 & 59.29 \\
\hline & Jog & Walk & Jumpshot & Layupshot & Pivot & Jumping & Running & Sprint \\
\hline
\end{tabular}

Figure 9. Confusion matrix produced by our recognition algorithm on basic basketball activities

\section{CONCLUSION}

In the paper, a new sensing system to record and recognize human movements in basketball has been presented. The new inertial measurement units are capable to record human movements at sport with high accelerometer ranges. The system has been employed to collect and analyze motion data in order to recognize basic activities in basketball. The proposed method is able to identify four moving types at different intensity (walking, jogging, running, and sprinting), as well as discriminate shooting executions. Moreover, the promising results prove the applicability of our proposed system within basketball activity monitoring.

Results are significant but with the addition of new features from accelerometer and including the gyroscope information the performance in activity recognition can be enhanced. In addition, a new dataset with more participants and, thus, with more heterogeneity of physical characteristics has been planned to be acquired.

\section{ACKNOWLEDGMENTS}

This work was supported in part by the Erasmus Mundus Joint Doctorate Programme in Interactive and Cognitive Environments.

Authors also would like to thank Dr. J.M. Padullés from Institut Nacional d'Educació Física de Catalunya for his valuable assistance in the design of the data collection plan.

\section{REFERENCES}

[1] Aggarwal, J., Ryoo, M.: Human Activity Analysis: A Review ACM Comput. Surv., ACM, 2011.

[2] Bulling, A., Blanke, U., Schiele, B. A.: Tutorial on Human Activity Recognition Using Body-worn Inertial Sensors. ACM Computing Surveys, 2014.

[3] Lara, O., Labrador, M. A .: Survey on Human Activity Recognition using Wearable Sensors. Communications Surveys Tutorials, IEEE, 2013.

[4] Kelly, D., Coughlan, G. F.; Green, B. S., Caulfield, B.: Automatic detection of collisions in elite level rugby union using a wearable sensing device. Sports Engineering, 2012. 
[5] Morris, D., Saponas, T. S.; Guillory, A., Kelner, I.: RecoFit: Using a Wearable Sensor to Find, Recognize, and Count Repetitive Exercises. Proceedings of the SIGCHI Conference on Human Factors in Computing Systems, ACM, 2014.

[6] Bächlin, M., Förster, K., Tröster, G.: SwimMaster: A Wearable Assistant for Swimmer. Proceedings of the 11th International Conference on Ubiquitous Computing, ACM, 2009.

[7] Harle, R., Taherian, S.; Pias, M.; Coulouris, G., Hopper, A., Cameron, J., Lasenby, J., Kuntze, G., Bezodis, I., Irwin, G., Kerwin, D.: Towards real-time profiling of sprints using wearable pressure sensors. Computer Communications, 2012.

[8] Ladha, C., Hammerla, N. Y.; Olivier, P., Plötz, T.: ClimbAX: Skill Assessment for Climbing Enthusiasts. Proceedings of the 2013 ACM International Joint Conference on Pervasive and Ubiquitous Computing, ACM, 2013.

[9] Velloso, E.; Bulling, A., Gellersen, H., Ugulino, W., Fuks, H.: Qualitative Activity Recognition of Weight Lifting Exercises. Proceedings of the 4th Augmented Human International Conference, ACM, 2013.

[10] Ghasemzadeh, H., Jafari, R.: Body sensor networks for baseball swing training: Coordination analysis of human movements using motion transcripts. Pervasive Computing and Communications Workshops (PERCOM Workshops), 2010 8th IEEE International Conference on, 2010.
[11] Rodríguez-Martín, D., Pérez-López, C., Samà, A., Cabestany, J., Català, A.: A Wearable Inertial Measurement Unit for Long-Term Monitoring in the Dependency Care Area. Sensors. 13, 14079-14104 (2013).

[12] Massé, F., Bourke, K., Chardonnens, J., Paraschiv-Ionescu, A., Aminian, K.: Suitability of commercial barometric pressure sensors to distinguish sitting and standing activities for wearable monitoring. Med. Eng. Phys. 36, 739-44 (2014).

[13] Zhou, S., Shan, Q., Fei, F., Li, W.J., Wu, P.C.K., Meng, B., Chan, C.K.H., Liou, J.Y.J.: Gesture recognition for interactive controllers using MEMS motion sensors. 2009 4th IEEE International Conference on Nano/Micro Engineered and Molecular Systems. pp. 935-940 (2009).

[14] Najafi, B., Aminian, K., Loew, F., Blanc, Y., Robert, P.A.: Measurement of stand-sit and sit-stand transitions using a miniature gyroscope and its application in fall risk evaluation in the elderly. IEEE Trans. Biomed. Eng. 49, 843-851 (2002).

[15] Barralon, P., Vuillerme, N., Noury, N.: Walk detection with a kinematic sensor: frequency and wavelet comparison. 28th Annu. Int. Conf. IEEE EMBS. 1, 1711-1714 (2006).

[16] Chang, C.-C., Lin, C.-J.: LIBSVM: A library for support vector machines. ACM Transactions on Intelligent Systems and Technology, 2011. 